\title{
(4S,5S)-2,2,4-Triethyl-5-methyl-1,3-dioxolane: A New Volatile Released by a Triatomine Bug
}

2010

Vol. 12, No. 24

$5601-5603$

\author{
C. R. Unelius, ${ }^{*, \dagger, \mid}$ \\ W. Francke ${ }^{\S}$ \\ B. Bohman, ${ }^{\dagger, \perp}$ M. G. Lorenzo, ${ }^{\ddagger}$ A. Tröger, ${ }^{\S}$ S. Franke, ${ }^{\S}$ and
}

School of Natural Sciences, Linnaeus University, SE-391 82 Kalmar, Sweden, Laboratório de Triatomíneos e Epidemiologia da Doença de Chagas, Centro de

Pesquisas René Rachou/FIOCRUZ, 30.190-002, Belo Horizonte, Minas Gerais, Brazil, and University of Hamburg, Organic Chemistry, Martin-Luther-King-Platz 6, D-20146 Hamburg, Germany

rikard.unelius@lnu.se

Received September 10, 2010

\section{ABSTRACT}

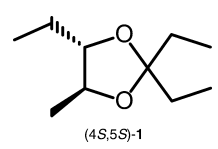

Adults of the triatomine bug Triatoma brasiliensis release 2,2,4-triethyl-5-methyl-1,3-dioxolane (1) as a mixture of the (4S,5S)- and (4R,5R)enantiomers in a ratio of $4: 1$. Among the volatile acetals identified from insects so far, this is the first example resulting from an intermolecular condensation of a carbonyl moiety and a diol substructure.

Blood-sucking bugs of the Triatominae subfamily transmit American trypanosomiasis, known as Chagas disease, in Latin America. ${ }^{1}$ Results of investigations on the chemical ecology of triatomine bugs have been reviewed by Cruz López et al. ${ }^{2}$ In Triatoma infestans (Klug 1834), analyses of volatiles of the so-called Brindley's glands, paired organs situated on the dorsolateral metathorax, or of head space volatiles collected over adult insects revealed the presence of carboxylic acids and corresponding alcohols, as well as their esters, as major components. ${ }^{2-5}$ The complete mixture, and isobutyric acid in particular, was reported to exhibit

\footnotetext{
$\dagger$ Linnaeus University.

Centro de Pesquisas René Rachou/FIOCRUZ.

$\S$ University of Hamburg.

" Present address: The Plant \& Food Research Institute of New Zealand, P.O.51 Lincoln, New Zealand.

${ }^{\perp}$ Present address: Research School of Chemistry and Research School of Biology, The Australian National University, Canberra ACT 0200, Australia.

(1) Dias, J.C P.; Silveira, A. C.; Schofield, C. J. Mem. Inst. Oswaldo Cruz 2002, 97, 603.

(2) Cruz López, L.; Malo, E. A.; Rojas, J. C.; Morgan, E. D. Med. Vet. Entomol. 2001, 15, 351.

(3) Fontán, A.; Audino, P. G.; Martinez, A.; Alzogaray, R. A.; Zerba, E. N.; Camps, F.; Cork, A. J. Med. Entomol. 2002, 39, 191.
}

repellent effects. Volatiles from another source, the paired metasternal glands that open to the ventral side of the metathorax, have been proposed to play a role in the mating behavior of triatomine bugs. ${ }^{6}$ The composition of the released blend differed considerably from that of Brindley's glands, showing 3-pentanone and 3-pentanol as main products. ${ }^{5}$

Another major component among the volatiles of the metasternal glands of Triatoma infestans remained unknown. ${ }^{7}$ During our recent investigations on another triatomine bug, T. brasiliensis (Neiva 1911), we encountered the same unknown compound. ${ }^{7}$ Its structure elucidation and synthesis are the subject of the present paper. Unfortunately, available amounts of the target compound were too small to obtain NMR spectra, and therefore, the structure assignment

(4) Guerenstein, P. G.; Guerin, P. M. Entomol. Exp. Appl. 2004, 111, 151.

(5) Manrique, G.; Vitta, A. C. R.; Ferreira, R. A.; Zani, C. L.; Unelius, C. R.; Lazzari, C. R.; Diotaiuti, L.; Lorenzo, M. G. J. Chem. Ecol. 2006, 32, 2035.

(6) Pontes, G. B.; Bohman, B.; Unelius, C. R.; Lorenzo, M. G. J. Chem. Ecol 2008, 34, 450.

(7) Vitta, A. C. R.; Bohman, B.; Unelius, C. R.; Lorenzo, M. G. J. Chem. Ecol. 2009, 35, 1212. 
had to be based on mass spectrometry (coupled with gas chromatography), microreactions, and independent synthesis.

The $70 \mathrm{eV}-\mathrm{EI}$ mass spectrum of the target compound is depicted in Figure 1.

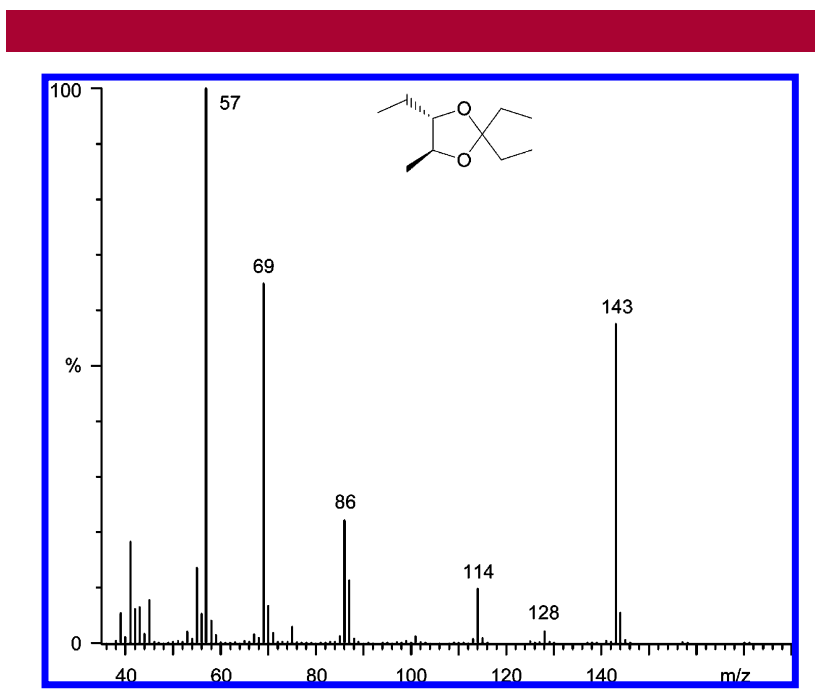

Figure 1. Mass spectrum (70 eV-EI) of (4S,5S)-2,2,4-triethyl-5methyl-1,3-dioxolane (trans-1).

The fragment with the highest mass recorded was represented by a signal at $\mathrm{m} / \mathrm{z}$ 143. High resolution mass spectrometry (GC/HR-MS) of the corresponding ion showed an atomic composition of $\mathrm{m} / \mathrm{z} 143=\mathrm{C}_{8} \mathrm{H}_{15} \mathrm{O}_{2}$. Chemical ionization analysis (GC/CI-MS) revealed the molecular mass of the natural product to be $\mathrm{M}^{+}=172$. As a result, its molecular formula proved to be either $\mathrm{C}_{10} \mathrm{H}_{20} \mathrm{O}_{2}(\mathrm{~m} / z, 143=$ M-ethyl) or $\mathrm{C}_{9} \mathrm{H}_{16} \mathrm{O}_{3}(\mathrm{~m} / \mathrm{z}, 143=\mathrm{M}$-formyl), exhibiting either one or two degrees of unsaturation, respectively. Because of its relatively low Kovats index of 1156 on a polar GC column (SupelcoWax-10), ${ }^{7}$ which indicated low polarity, the latter atomic composition was regarded less likely. As a consequence, the structure of the unknown was expected either to be cyclic or to contain a carbonyl group or a CC double bond. The latter case was ruled out because the compound could not be catalytically hydrogenated. ${ }^{8}$ The intense signal at $\mathrm{m} / \mathrm{z} 143$ indicated a rather stable fragment, supposedly due to the formation of an oxonium ion. Corresponding structures are typically formed upon $\alpha$-cleavage from secondary and tertiary alcohols or ketones as well as from ethers and acetals (including cyclic structures). ${ }^{9,10}$ As acetylation ${ }^{8}$ failed and because of the low Kovats index of the compound, alcohol structures were considered less likely.

Based on the analytical information obtained so far, the natural product was hypothesized to be a cyclic acetal. Because of the large amounts of 3-pentanone present in the metasternal glands and the abundant signal at $\mathrm{m} / \mathrm{z} 143=$ $\mathrm{M}^{+}$-ethyl in the mass spectrum of the target compound, the

(8) Attygalle, A. B. In Methods in Chemical Ecology 2; Millar, J. G. Haynes, K. E., Eds.; Kluwer: New York, 1998; pp 207-294.

(9) Francke, W.; Kitching, W. Curr. Org. Chem. 2001, 5, 233.

(10) Marshall, J. T. B.; Williams, D. H. Tetrahedron 1967, 23, 321. parent carbonyl compound involved in the formation of the target compound was regarded to be 3-pentanone. Consequently, 2,2-diethyl-1,3-dioxanes or 2,2-diethyl-1,3-dioxolanes, carrying the remaining carbons as alkyl substituents, were considered to be suitable candidates. Finally, two less abundant signals in the mass spectrum, detected at $\mathrm{m} / \mathrm{z} 128$ $\left(\mathrm{C}_{8} \mathrm{H}_{16} \mathrm{O}\right.$ upon HR-MS) and $\mathrm{m} / z, 114\left(\mathrm{C}_{7} \mathrm{H}_{14} \mathrm{O}\right.$ upon HR-MS) (Figure 1), proved to represent key fragments decisive for structure elucidation. Earlier it had been shown that upon electron impact 4,5-dialkyl-1,3-dioxolanes extrude carbonyl fragments consisting of an alkyl substituent, the corresponding ring-carbon, and the adjacent oxygen.${ }^{11}$ In the present case, this was suggested to be acetaldehyde giving rise to $\mathrm{M}^{+}-44=m / z, 128$ and acetone or propanal producing $\mathbf{M}^{+}$$58=\mathrm{m} / \mathrm{z}$ 114. Consequently, the target compound was considered to be either 2,2-diethyl-4,4,5-trimethyl-1,3-dioxolane or 2,2,4-triethyl-5-methyl-1,3-dioxolane.

The former compound, prepared upon boron trifluoride catalyzed reaction ${ }^{12}$ of 3-pentanone and 2,2,3-trimethyloxirane, yielded a mass spectrum similar to that of the natural product (see Supporting Information); however, the gas chromatographic retention time did not match. We, therefore, synthesized both racemic cis-2,2,4-triethyl-5-methyl-1,3dioxolane (cis-1) and its trans-isomer. Boron trifluoride catalyzed reaction of 3-pentanone with either trans-2-ethyl3-methyloxirane (trans-2) (obtained from (E)-2-pentene upon epoxidation with $m$-chloroperbenzoic acid) or cis-2-ethyl3-methyloxirane (cis-2) (from (Z)-2-pentene) produced the desired dioxolanes in good yields (Figure 2). NMR data of

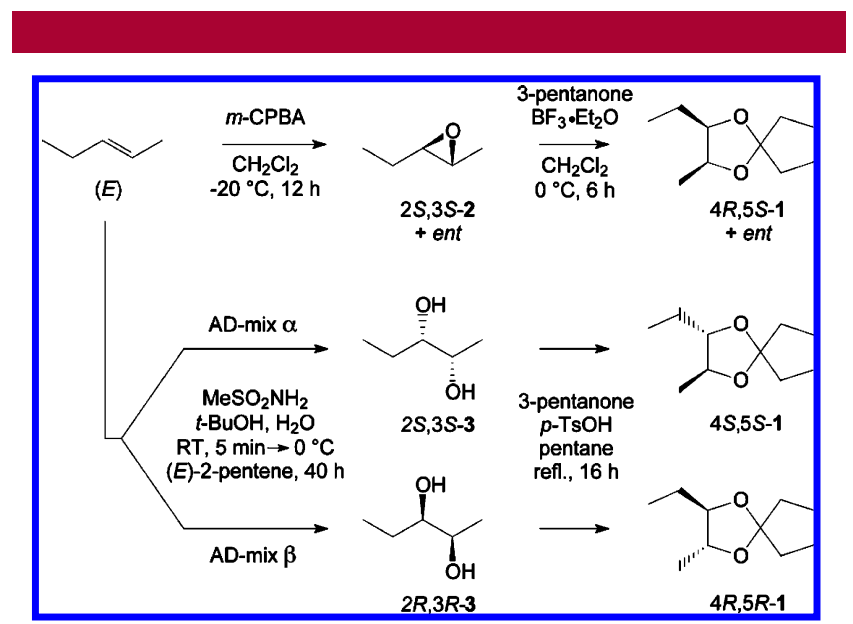

Figure 2. Synthesis of racemic and optically active 2,2,4-triethyl5-methyl-1,3-dioxolanes.

the products were as expected. Upon gas chromatography, the diastereomers were well resolved $(\alpha=\operatorname{tr}$ cis/tr trans $=$ 1.10 on CP-Sil 5 and 1.02 on FFAP). While the mass spectra of both isomers matched that of the natural product, the gas chromatographic retention time of trans-2,2,4-triethyl-5methyl-1,3-dioxolane coincided with that of the target

(11) Friedel, R. A.; Sharkey, A. G., Jr. Anal. Chem. 1956, $28,940$.

(12) Blackett, B. N.; Coxon, J. M.; Hartshorn, M. P.; Lewis, A. J. Tetrahedron 1970, 26, 1311. 
compound. However, a close inspection of our GC/MS data revealed the presence of tiny amounts of the later eluting (and higher boiling ${ }^{13}$ ) cis-isomer (see Supporting Information).

As a final step, the enantiomeric composition of the naturally occurring trans-2,2,4-triethyl-5-methyl-1,3-dioxolane had to be determined by employing enantioselective gas chromatography. For the syntheses of optically active reference samples, $(E)$-2-pentene was used as the starting material. A Sharpless bishydroxylation approach ${ }^{14}$ applying AD-mix $\alpha$ or AD-mix $\beta$ yielded ( $2 S, 3 S)$-2,3-pentanediol ( $S$ 3 ) or its enantiomer, respectively (Figure 2). The NMR spectra of the obtained diols were in accord with data reported in the literature. ${ }^{15}$

Upon enantioselective gas chromatography using modified cyclodextrins, the enantiomers of trans-2,2,4-triethyl-5methyl-1,3-dioxolane, prepared through an acid-catalyzed reaction of 3-pentanone with optically active syn-2,3pentanediols, were baseline separated.

The synthetic trans-configured 1,3-dioxolanes showed an enantiomeric excess (ee) of $92 \%$ for the $(4 S, 5 S)$-stereoisomer and $90 \%$ for its earlier eluting $(4 R, 5 R)$-enantiomer. Comparison of mass spectra and retention times (coinjection) of the synthetic reference compounds with corresponding data of the natural product revealed the latter to be a mixture of $(4 S, 5 S)$ - and $(4 R, 5 R)-2,2,4$-triethyl-5-methyl-1,3-dioxolane in a ratio of $4: 1$ (Figure 3 ). Due to the particularly small amounts of cis-1 and the interference with other volatiles

(13) van Risseghem, H. Bull. Soc. Chim. France 1960, 27, 1192.

(14) Sharpless, K. B.; Amberg, W.; Bennani, Y. L.; Crispino, G. A.; Hartung, J.; Jeong, K.-S.; Kwong, H.-L.; Morikawa, K.; Wang, Z.-M.; Xu, D.; Zhang, X.-L. J. Org. Chem. 1992, 57, 2768.

(15) Enders, D.; Nakai, S. Chem. Ber. 1991, 124, 219.

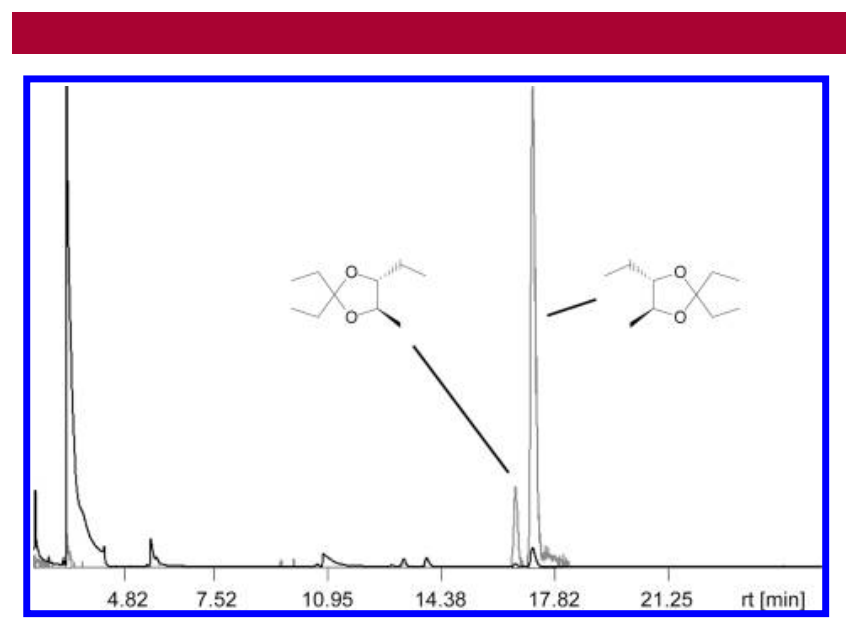

Figure 3. Chromatogram of an extract of metasternal glands of $T$. brasiliensis using enantioselective GC/MS. TIC in black and ion chromatogram for $m / z, 143$ in gray. Tiny amounts of the later eluting cis-isomer present in the glands did not produce a visible signal in this GC (conditions: ref 7).

present in the glands, it was impossible to determine the enantiomeric composition of this isomer.

Investigations concerning behavior-mediating activities of the new Triatoma compounds are underway.

Supporting Information Available: Experimental procedures, analytical data, and copies of ${ }^{1} \mathrm{H}$ and ${ }^{13} \mathrm{C}$ NMR spectra for cis-1 and trans-1 and gas chromatograms of natural extracts. This material is available free of charge via the Internet at http://pubs.acs.org.

OL102165Q 